\title{
Ferropenia y Anemia Ferropriva en Lactantes y Preescolares Normales
}

\author{
T.M. Iván Palomo G.; T.M. Brunilda Gutièrrez C.; T.M. Mima Guerra R.; \\ Enf. Marta de la Fuente L. 2 Sr. Mario Pino F. 3
}

\author{
Iron Deficiency and Iron Deficiency Anemia in Well Nourished Infants \\ adn Children.
}

Twohundred and seventeen infants and onehundred and fourtynine children well nourished of low socio-economical condtion were studied for iron deficiency. Incidence of iron deficiency was greater in infants $(38,4 \%)$ than in children $(17,2 \%$. Equally, the percentage of Lron deficiency anemia was higher although in a lower grade in infants $(33 \%)$ than in children $(8,5 \%)$.

El Hierro es un elemento esencial para todo organismo vivo. Participa en numerosas reacciones de oxido reducción por poseer la particularjdad de captar y ganar electrones en forma reversible. Lo encontramos participando en diversos proeesos vitales a través de la Hemoglobina $(70 \%$ del Fe del adulto), Mioglobina ( \pm 4.0\%), Depósitos $( \pm 25 \%)$, y un pequeño pero importante porcentaje $(<1 \%)$ formando parte de numerosas enzimas o cofactores. Esta última fracción contribuye a mantener la integridad de los epitelios, las

1 Tecnólogos Médicos, sección Hematología, labotatorio Clínico Central, Hospital Regional de Talca.

2 Enfermera, Consultorio Arturo Prat, Universidad de Talca.

${ }^{3}$ Profesor Bicestadística, Universidad de Talca. funciones inmunológicas y la salud física y psíquica en general. ${ }^{3}$

Asi, la carencia de Fe. afecta en mayor o menor grado a todas las células de un organismo vivo y se traducirá por lo tanto en una enfermedad sistemática que puede comprometer el sisteina Hematopoyético, metabolismo general, sistema muscular, sistema nervioso central, piel y mucosas, sistema digestivo y sisterma inmunológi$\mathrm{CO}^{2-3}$

La edad pediátrica es una etapa de continuo déficit de hierro y en ella el período de lactante es de especial vulnerabilidad, por el rápido crecimiento y la alimentación básicamente láctea, que carece de $\mathrm{Fe}^{4}$.

La alta prevalencia de este déficit ha sido bien documentada en diferentes lugares, haciendo de 
éste, tal vez la deficiencia nutricional más difundida en el mundo ${ }^{5}$.

En la edad pediátrica, y sobre todo en el lactante debido a los cambios fisiológicos que en relación al $\mathrm{Fe}$ ocurren, ha sido difícil determinar cuando se está en presencia de un niño normal o anornal en este aspecto.

Para solucionar este problema, centros especializados han realizado en nir̃os previamente suplementados con hierro, estudios de Laboratorio en que han determinado diferentes parámetros. Tomando los límites inferiores de normalidad para Hemoglobina (Hb) y Saturación de transferrina obtenidos en estos estudios. (ver comentario), es posible catalogar los casos estudiados como ferropénicos, anérnicos o normales.

La incidencia de Ferropenia y Anemia ferropriva varía de un país a otro e incluso dentro de un mismo país. Esto se podría explicar por la diversidad de métodos de laboratorio, criterio diagnóstico de Anemia y Ferropenia adoptado por los investigadores 6 yabría que agregar la variación que representa el grupo humano estudiado en lo que respecta a nivel socio-econónico y altura sobre el nivel del mar?

En nuestro medio no existía información sobre esta matería, siendo esta una de las razones para realizar este estudio, con que tuvo el propósito de determinar los valores promedios de algunos parámetros en la población estudiada; conocer la incidencia de Ferropeniá y Anemia. Ferropriva y relacionar algunos de los parámetros entre sí y con la edad.

\section{MATERIAL Y METODO}

El grupo estudiado estuvo formado por 217 lactantes y 149 preescolares controlados en el Consultorio "Arturo Prat" de la ciudad de Talca, cuya población beneficiaria está constituida aproximadamente por un $60 \%$ de familias de nivel socio-económico bajo ${ }^{8}$.

Para ser incluido en el estudio se exigió en cada caso las condiciones de asistir regularmente a los controles de salud; liaber sido catalogado como niño bien nutrido según relación Peso/Edad \pm 1 DS, usando tablas de Sempé*; no haber consultado por patología aguda en los últimos 10 días, ni ser portador de una patología

* Estudio semilongitudinal de M. Sempé seguido por M.P. Roy y Padrón (1971). Tabla distribuida por el Servicio de Salud local y el Ministerio de Salud de Chile. crónica; haber tenido un peso de nacimiento superior a $2.500 \mathrm{~g}$.

En el grupo estudiado se encontró un peso de nacimiento promedio de $3.380 \mathrm{~g} \pm 2 \mathrm{DS}=880 \mathrm{~g}$.

Las muestras fueron tomadas en ayunas en la Sección de Hematología del Laboratorio Central. En la recolección y en el procesamiento de las muestras se utilizó material libre de $\mathrm{Fe}$.

Los parámetros considerados fueron el Hematocrito $(\mathrm{Ht})$, la concentración de hemoglobina $(\mathrm{Hb})$, Ferremia (Fe) y Saturación de transferrina (TIBC).

El hematocrito se obtuvo con técnica de micro-hematocrito.

La hemoglobina se determinó con el Método de cjanuro de Hemiglobina; calibrado con estándar proporcionado por' el Laboratorio de Referencia del Instituto de Salud Pública. Este método es periódicamente evaluado por el Laboratorio de Referencia, además se realizaron curvas de calibración cada 2 meses. Se ha obtenido 'en condiciones de rutina, un coeficiente de variación (c.v.): $0,76 \%$ y un coeficiente de corre. lación (r): 0.9998.

La Ferremia y TIBC se midieron con los Kits Boehringer Mannheim, número de catálogos: $\mathrm{Fe}$ 123222 y TIBC 125806. En condiciones de rutina se mantiene bajo control (suero control: Precipath $U$ cat. $N^{\circ} 171778$, Boehringer Mannheim).

En las determinaciones de $\mathrm{Hb}$ y $\mathrm{Fe}-\mathrm{TIBC}$, se utilizó un Espectro-fotómetro Shidmadzu U.V. 120-02, al cual se controla periódicamente la calibración de la longitud de onda y linealidad fotometrica.

\section{RESULTADOS}

Antes de presentar los datos obtenidos, creemos necesario explicar que los casos correspondientes al grupo de 18-24 meses fueron difíciles de conseguir debido a que en esa edad, el lactante no está obligado a concurrir al control de salud, lo que explica el menor numero de casos.

No se hizo tabulación por sexo, pues trabajos anteriores ${ }^{9}$ muestran que no habria diferencias hasta aproximadamente los 11 años.

En la Tabla 1, se muestra la evolución de los parámetros $\mathrm{Hto}, \mathrm{Hb}, \mathrm{Fe}$ y $\mathrm{TIBC}$, en diferentes edades del lactante y preescolar.

Los valores medios de Hto. aumentan desde $32,9 \%$ en el grupo de 3 a 6 meses hasta $36,5 \%$ en el grupo de 4 a 6 años. Paralelamente aumentó la $\mathrm{Hb}$ desde $10,8 \mathrm{~g} / \mathrm{dl}$ hasta $12,3 \mathrm{~g} / \mathrm{dl}$.

El Fe sérico y la saturación de transferrina aumentaron desde 47,9 ugs/dl y 13,7\% a 82,2 ugs/dl y $26,6 \%$ respectivamente. Se presenta un pequeño descenso en el grupo de 18 a 24 meses. 
Tabla 1.

Valores Medios de Hto., Fe., y Saturación de Transferrina a Distintos Grupos Etarios

\begin{tabular}{|c|c|c|c|c|c|c|c|c|c|c|}
\hline \multirow[t]{2}{*}{ EDAD } & \multicolumn{3}{|c|}{ Hto $(\%)$} & \multicolumn{2}{|c|}{$\mathrm{Hb}(\mathbf{g} / \mathbf{d} \mathbf{l})$} & \multicolumn{3}{|c|}{$\mathrm{Fe}(\mathrm{ugr} / \mathrm{dl})$} & \multicolumn{2}{|c|}{$\begin{array}{c}\text { Sat de } \\
\text { Transferrina }\end{array}$} \\
\hline & n & $\overline{\mathbf{x}}$ & \pm D.S. & $\overline{\mathrm{x}}$ & $\pm \mathrm{D} . \mathrm{S}$. & $\mathbf{n}$ & $\overline{\mathbf{x}}$ & \pm D.S. & $\overline{\mathbf{x}}$ & \pm D.S. \\
\hline $3-6 m$ & 40 & 32,9 & 2.3 & 10.8 & 1.0 & 40 & 47.9 & 26.0 & 13.7 & 7.0 \\
\hline$+6-12 \mathrm{~m}$. & 48 & 34.3 & 2.5 & 11.2 & 1.2 & 51 & 52.7 & 23.2 & 14.5 & 6.9 \\
\hline$+12-18 \mathrm{~m}$ & 63 & 35.0 & 2.6 & 11.2 & 1.1 & 66 & 53.6 & 25.2 & 13.7 & 9.1 \\
\hline$+18-24 m$ & 37 & 34.9 & 3.7 & 11.4 & 1.6 & 32 & 48.9 & 35.4 & 12.5 & 8.8 \\
\hline$+2-4 a$ & 68 & 35.9 & 2.4 & 11.9 & 1.1 & 60 & 77.0 & 36.2 & 21.7 & 10.4 \\
\hline$+4-6 a$. & 75 & 36.5 & 2.3 & 12.3 & 0.9 & 66 & 82.2 & 32.9 & 26.6 & 10.0 \\
\hline
\end{tabular}

Al analizar la relación existente entre $\mathrm{Hb}, \mathrm{Hto}$ y CHCM encontramos que en ningún lactante la Hb era mayor que $14 \mathrm{~g} / \mathrm{dl}$ y en ningún niño de 4 a 6 años se encontró $\mathrm{Hb}$ menor que $10 \mathrm{~g} / \mathrm{dl}$ y concentraciones de $\mathrm{Hb}$ corpuscular media (CHCM) inferiores a $30 \%$.

Los valores mínimos de los rangos de Hto para Hb de $11 \mathrm{~g} / \mathrm{d}$ fluctuaron entre 31 y $33 \%$, sin presentar obviamente diferencias significativas entre uno y otro grupo etario.

Todos los casos con $\mathrm{Hb}$ mayor que $11 \mathrm{~g} / \mathrm{dl}$ presentaron CHCM superior a $30 \%$, apareciendo progresivamente la desaturación a medida que baja el valor de $\mathrm{Hb}$.

Todos los easos con CHCM menor que $30 \%$ correspondieron a $\mathrm{Hb}$ bajo $11 \mathrm{~g} / \mathrm{dl}$. Esta situación se presentó en $12,5 \%$ y $15,7 \%$ en los lactantes de 3 a 6 meses y de más de 6 a 24 meses respectivamente y $5,8 \%$ en los preescolares de 2 a 4 años.

La Tabla 2 muestra la relación obtenida entre $\mathrm{Hb}$ y Saturación de transferrina en los diferentes grupos etarios, para ello se registra el promedio de $\mathrm{Hb}$ que se obtuvo en cada rango de Saturación de transferrina. Se puede observar una correlación positiva o con esa tendencia desde 1 a 6 ẵos.

Para Ja Tabla 3, se consideró Anemia, las Hb menor de $11 \mathrm{~g} / \mathrm{dl}$ y Ferropenia; Saturación de transferriua inferior a 10 y $15 \%$ para lactantes y preescolares respectivamente.

Tabla 2.

Correlación entre Hb. y Saturación de Transferrina a distintas edades

\begin{tabular}{|c|c|c|c|c|c|c|c|c|}
\hline \multirow{2}{*}{ EDAD } & \multirow{2}{*}{$\mathbf{H b}$} & \multicolumn{5}{|c|}{ Saturación de Transferrina } & \multirow{2}{*}{$\begin{array}{c}\text { Coeficiente } \\
\text { de } \\
\text { Correlación }\end{array}$} & \multirow{2}{*}{$\begin{array}{l}\text { Significancia } \\
\text { Estadística }\end{array}$} \\
\hline & & $<10$ & $10-15$ & $15-20$ & $20-25$ & $>25$ & & \\
\hline $6-12 \mathrm{~m}$ & $\underset{\substack{\mathbf{X} \\
\text { rango }}}{n}$ & $\begin{array}{c}11.2 \\
8.6-12.9 \\
12\end{array}$ & $\begin{array}{c}11.3 \\
9.0-13.1 \\
16\end{array}$ & $\begin{array}{c}11.2 \\
8.8-13.3 \\
6\end{array}$ & $\begin{array}{c}12.3 \\
12.1-12.5 \\
5\end{array}$ & $\begin{array}{c}11.1 \\
10.5-11.6 \\
2\end{array}$ & 0.34 & No significativo \\
\hline$+12-18 \mathrm{~m}$ & $\begin{array}{c}\vec{X} \\
\text { rango } \\
n .\end{array}$ & $\begin{array}{c}10.9 \\
8.8-13.9 \\
22\end{array}$ & $\begin{array}{c}11.4 \\
9.6-13.4 \\
13\end{array}$ & $\begin{array}{c}11.5 \\
8.9-13 \\
12\end{array}$ & $\begin{array}{c}12 \\
11.3-12.3 \\
4\end{array}$ & $\begin{array}{c}11.5 \\
10.7-12.6 \\
5\end{array}$ & 0.75 & Significativo \\
\hline$+18-24 \mathrm{~m}$ & $\begin{array}{c}\bar{X} \\
\text { rango } \\
n\end{array}$ & $\begin{array}{c}9.9 \\
8.6-11.4 \\
13\end{array}$ & $\begin{array}{c}12.6 \\
11.1-13.9 \\
6\end{array}$ & $\begin{array}{c}10.8 \\
10.7-10.9 \\
2\end{array}$ & $\begin{array}{c}12.3 \\
12.1-12.5 \\
2\end{array}$ & $\begin{array}{c}12.4 \\
12.0-13.0 \\
4\end{array}$ & 0.57 & Significativo \\
\hline$+2-4 a$ & $\begin{array}{c}\bar{x} \\
\text { rango } \\
n\end{array}$ & $\begin{array}{c}11.0 \\
8.5-12.6 \\
12\end{array}$ & $\begin{array}{c}12.7 \\
12.6-12.8 \\
2\end{array}$ & $\begin{array}{c}12.0 \\
11.0-13.7 \\
9\end{array}$ & $\begin{array}{c}12.0 \\
11.1-13.0 \\
12\end{array}$ & $\begin{array}{c}12.5 \\
14.1-14.0 \\
20\end{array}$ & 0.46 & $\begin{array}{l}\text { Tiende a ser } \\
\text { significativo }\end{array}$ \\
\hline$+4-6 a$ & $\underset{\substack{\mathbf{x} \\
\text { rango }}}{\bar{n}}$ & $\begin{array}{c}11.3 \\
10.2-12.5 \\
4\end{array}$ & $\begin{array}{c}11.4 \\
10.8-11.8 \\
3\end{array}$ & $\begin{array}{c}12.2 \\
11.4-13.2 \\
9\end{array}$ & $\begin{array}{c}12.1 \\
11.0-13.9 \\
11\end{array}$ & $\begin{array}{c}12.5 \\
11.0-13.5 \\
37\end{array}$ & 0.93 & Significativo \\
\hline
\end{tabular}


Tabla 3.

Incidencia de Fertopenia y Anemia Ferropriva a distintas edades.

EDAD

$\begin{array}{lcccc} & \mathrm{n} & \%<11 \mathrm{~g} / \mathrm{dl} & \mathrm{n} & \% \\ 3-6 \mathrm{~m} & 40 & 57.5 & 40 & 32.5 \\ +6-12 \mathrm{~m} . & 48 & 33.3 & 51 & 27.5 \\ +12-18 \mathrm{~m} . & 63 & 33.3 & 66 & 40.9 \\ +18-24 \mathrm{~m} . & 37 & 32.4 & 32 & 46.8 \\ +2-4 \mathrm{a} & 68 & 10.3 & 60 & 25.0 \\ +4-6 \mathrm{a} & 75 & 6.6 & 66 & 10.6\end{array}$

* Sat de Transferrina: Lact. $<10 \%$ y Preesc. $<15 \%$ ** : $12.5 \%$ de casos con $\mathrm{Hb}<10 \mathrm{grd} / \mathrm{d} 1$.

Se observó que el grupo 3-6 meses fue el que presentó mayor porcentaje de anemia $(57,5 \%)$. (Este porcentaje en realidad es menor si consideramos que estamos abarcando la edad en que se presenta la "Anemia Fisiológica". Así en este mismo grupo etario el porcentaje con $\mathrm{Hb}$ menor de $10 \mathrm{~g} / \mathrm{dl}$ es de $12,5 \%$ ). Esta frecuencia decrece progresivamente hasta un $32,4 \%$ al término del período de lactante y más aún en los preescolares $(10,3 \%$ y $6,6 \%)$.

Las Ferropenias más marcadas, se observaron en lactantes y con mayor frecuencia entre los 12 y 24 meses $(40,9$ y $46,8 \%$ ). Disminuyó significativamente en los preescolares $(25,0$ y $10,6 \%)$.

\section{COMENTARIO}

Los valores medios encontrados por nosotros, son inferiores a los encontrados por otros autores $^{10}$ que también estudjaron niroos bien nutridos.

La literatura ${ }^{5-1]}$ plantea el grupo etario que va de los 6 a 18 meses como el más propenso a sufrir la enfermedad ferropriva. Esto se explicaria porque los depósitos de hierro alcanzan su etapa crítica a los 6 meses en el recién nacido de término $y$ al no recibir un aporte adecuado, pasará secuencialmente por los estados de Depleción de Hierro, Eritropoyesis Ferropriva y finalmente Anemia Hipocroma Ferropriva.

En el grupo de 3 a 6 meses los valores más bajos de $\mathrm{Hto}, \mathrm{Hb}$, se podrian explicar debido a que en estos casos se incluyen lactantes en etapa de "Anemia Fisiológica".

Los valores de $\mathrm{Fe}$ sérico en el mismo grupo también son bajos lo que estaría indjcando una ferropenia precoz. Esto podría explicarse en parte por que un $60,7 \%$ de los casos de 3 a 6 meses, tuvo menos de 3 meses de lactancia natural. Este antecedente cobra importancia, si se recuerda que la biodisponibilidad del $\mathrm{Fe}$ en la leche materna es $50 \%$ y en la de vaca no modificada sólo $0,5 \%$.

Entre los 6 y 24 meses los cambios en Hto y $\mathrm{Hb}$ no son significativos, obteniéndose como promedjos $34.5 \%$ y $11.3 \mathrm{~g} /$ dl respectivamente.

En los preescolares, se obtuvo promedios de $\mathrm{Hto}, \mathrm{Hb}, \mathrm{Fe}$, y Sat. de Transferrina significativamente más altos al igual que otros auto.

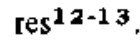

Encontramos una correlación positiva entre $\mathrm{Hb}$ y $\mathrm{CHCM}$; aumentando el número de casos con CHCM inferior a $30 \%$, a medida que disminuye la concentración de $\mathrm{Hb}$.

Con una CHCM bajo $30 \%$ se considera una Anemia Hipocroma ${ }^{14}$. Estos mismos autores también encontraron una cortelación positiva entre $\mathrm{Hb}$ y CHCM.

Mandujano ${ }^{15}$, encontró un $57,6 \%$ de lactantes con $\mathrm{CHCM} 30 \%$, porcentaje superior al nuestro $(30 \%)$.

Sümes y Cols. ${ }^{16}$, plantean que incluso en una población no deficiente en fierro, la $\mathrm{Hb}$ se comporta como una variable dependiente de la Sat. de Transferrina hasta los 12 meses de edad, y no detectable o poco significativa en el resto de los grupos etarios. Margozzini y Cols ${ }^{10}$, tarnbién encontraron esta correlación en una población con 14.3\% de Anemia. Nosotros no obtuvimos una correlación positiva entre los 6 y 12 meses. Creemos que deberia estudiarse con un mayor número de casos.

Para la tabulación de datos se usaron criterios de normalidad encontrados por autores extranje$\operatorname{ros}^{9-17 \cdot 18}$, quienes minimizaron la influencia del déficit de hierro y otras causas comunes de anemia, como fuentes de error en estudios controlados de niños con hierro suplementario y por el uso de pruebas de Laboratorio de alta tecnología. Así, fueron considerados límites inferiores de normalidad: $\mathrm{Hb}: 11 \mathrm{~g} / \mathrm{dl}$, Fe sérico: $30 \mu \mathrm{g} / \mathrm{dl}$ y Saturación de transferrina: 10 y $15 \%$ para lactantes sobre 5 meses y preescolares respectivamente.

En la población estudiada se encontró un $33.0 \%$ y $8.5 \%$ de Anemia Ferropriva ( $\mathrm{Hb}$ menor que $11 \mathrm{~g} / \mathrm{d}$ l) y un $38.4 \%$ y $17.2 \%$ de Ferropenia (Sat. de Transferrina - menor que 10\% lactantes y $15 \%$ preescolares) en lactantes mayores de 6 meses y preescolares respectivamente.

Basados en el criterio de $\operatorname{Cook}^{19}$, el grupo de 6 a 24 meses se encontraría en Eritropoyesis ferropriva, el de 2 a 4 años en Depleción de Hierro y por último el de 4 a 6 años tiende a la normalidad (normal: aproximadamente $2.5 \%$ de los casos con $\mathrm{Hb}$ menor que $1 \mathrm{lg} / \mathrm{dl}$. Como promedio se obtuvo un $33.0 \%$ y 8.5 de $\mathrm{Hb}$ inferior a $11 \mathrm{~g} / \mathrm{dl}$, en lactantes y preescolares 
respectivamente. Estas cifras indicarian que la población lactante se encontratía en un estado de Depjeción de Hierro.

Otros autores, con este mismo criterio de normalidad para $\mathrm{Hb}$, han encontrado porcentajes de Anemia que van del 15 al 40\% 10-12-15, para lactantes y $9.2 \%^{12}$ para preescolares. Estos porcentajes serian semejantes a los nuestros. En países Sudamericanos las cifras fluctúan entre 15 y $50 \% 5$.

Los trabajos chilenos revisados ${ }^{\text {0-12-13.15, }}$ han considerado $15 \%$ como límite inferior de normalidad para Sat. de Transferrina, lo cual dificulta las comparaciones en cuanto a incidencia de Ferropenja, debido a que nosotros consideramos esta cifra sólo para preescolares y $10 \%$ para lactantes. A pesar de ello se puede concluir que la frecuencia de Ferropenia encontrada por nosotros en lactantes (38.4\%), es inferior a la encontrada por Taboada y Cols. quienes obtuvieron aproximadamente un $60 \%$ de casos con Sat. de Transferrina menor que $10 \%^{1}$, cifra que se repite en los otros autores ${ }^{10 \cdot 12-13}$.

Hacemos notar que en los preescolares disminuye progresivamente la frecuencia de Ferropenia; $25.0 \%$ y $10.6 \%$ en los grupos de 2 a 4 años y 4 a 6 años respectivamente. Se obtiene como promedio de Ferropenia para preescolares, un $17.2 \%$.

Las cifras presentadas en la Tabla 3, muestran una alta frecuencia de Anemia y Ferropenia en lactantes. Además sin variar las condiciones socioeconómicas, disminuyen espontáneamente estos porcentajes a medida que salen de este período, lo cual podría explicarse por una dis. minución en el ritmo de crecimiento, lo que a su vez acarrea una menor necesidad de nutrientes ${ }^{15}$. Se sabe que el metabolismo del Fe en el lactante es precario, debido fundamentalmente a la baja reserva al nacer, gran demanda por crecimiento y bajo aporte dietético. Teniendo en cuenta lo anterior, se puede comprender que un individuo de medio socioeconómico bajo, sufrirá las con. secuencias de la suma de ellas con mayor intensi$\mathrm{dad}^{14}$.

Esta alta prevalencia de Ferropenia y Anemia Ferropriva, debe ser considerado un problema de salud pública y por lo tanto se deben agotar los esfuerzos en su prevención. El ideal sería a través del consumo de una dieta adecuada, pero en el caso del lactante es posible que incluso las mejores dietas $y$ en el mejor ambiente no logren prevenir la carencia de Hierro en todos los ca. $\operatorname{sos}^{11}$.

Como la alternativa más práctica y eficiente, expertos de OMS plantean la suplementación de alimentos con $\mathrm{Fe}^{20}$.
En nuestro país, distintos grupos hacen esfuerzos en lograr la mejor fórmula alimenticia fortificada con $\mathrm{Fe}^{21-22}$.

\section{RESUMEN}

Se estudiaron 217 lactantes y 149 preescolares, bien nutridos de bajo nivel socio-económico. La incidencia de Fertopenia fue mayor en lactantes $(38,4 \%)$ que en preescolares $(17,2 \%)$. Igualmente el porcentaje de Anemia Ferropriva fue mayor aunque en menor grado en lactantes $(33,0 \%)$ que en preescolares $(8,5 \%)$.

\section{AGRADECIMIENTOS A.:}

Dr. Hernán Taboada

Hematólogo Hospital Josetina Martínez, Santiago.

Q.F. Lola Ramos I.

Jefe Laboratorio Clínico Central, Hospital Regional Talca.

\section{REFERENCIAS}

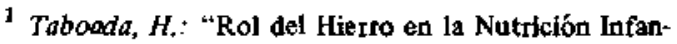
tíl", 1 parte Rev. Chil. Ped., 54: 47, 1983.

2 Grebe, G.; Lira, P.; Faradi, A.: "Carencia de Hierro de Origen Nutricional", Rev. Méd. Ch. 104: 954, 1976.

3 Taboada, H.: "Rol del Hierro en la Nutrición Infantil", II parte, Rev, Chil. Ped., 54: 132, 1983.

- Rtos, E.L.: "Metabolisma del Hierro en el Hombre", Apartado Docente, No, 50, 1977

5 Joint FAO/WHO.: Expert group on Requirements of Ascorbic Acid, Vitamin D., Vatamin B12, Folate and Iron. Wld. Hlth. Org. Techn. Rep. Ser. No 452, 1970.

"Daiber, A: "El Glóbulo Rojo y sus alteraciones", Stgo. 1980.

7 Grupo de Estudio de la OMS sobre Anemig Femoprivo.: "Anemias Ferropénicas". Informe Técnico No $182,1959$.

- Rojas, E.A.: "Estudio población de alto rie sgo". Lniversidad de Talca, 1982.

" Dallman, P.; Sïmes, M.: "Percentile curves for hemoglobin and red cell volume in infancy and chilthood". J. Pediat., 94: 26, 1979.

10 Margozini, J.; Bravo, M,; Lanzkowsky, P,; Vallente, S.; Rosales, E.; Urteaga, C.; Vargas, S.: López, I.; Covarrubias, E.; Puratic, O.; Arauco, G.: "La corencia de Fierro en el Lactante considerado Eutrófico, Area Hospitalaria Norte de Santiago". Rev. Chil. Ped. 48: 9, 1972.

1 Stekel, A.: Otivares, M.; Lopez, I.: Chadud, P.; Castaño, G.: "Deficiencia de Hierro y enriquecimiento de Alimentos", Rev. Chil Ped. 44: 447, 1973.

12 Winter, A.; Arteaga, A.; Galofia, A.: Maiz A.: "Prevalencia de Anemia Ferropriva y Deficiencia de Hjerro en una Población Materno Infantil del Area Sur Oriente de Santiago de Chile". 1970, Rev. Chil. Ped. 45: 53, 1974.

13 Arteaga, A.; Galofra, A.; Matz, A.; y Cols.: "Estudio Nutricional de una población infantil del Area Sur Oriente de Santiago (1970)". Rev. Chï. 102: 387 , 1974 . 
14 Winter, A.; Taboada, H.; Ducos, $R_{.:}$Norambuena, $N$,; Vera, S.; Delgado. Cr.. "Incidencia de Anemia y Ferropenia en el Lactante Chileno". Rev. Chil. Ped. No 11-12: 1035, 1970.

15 Mandujano. Y. y Cols.: "Carencia de Fierro en el Lactante", Tesis Fac. Quím. Farm.; Universidad de Chile, 1970 .

16 Sümes, M.: Saarinen, $U_{\text {.; }}$ Dollman, $P_{\text {.: }}$ "Relationship between hemoglobin concentration and transferrin saturation in iron sufficient infart". Am. J. Clin. Nutr. 32: 2295, 1979.

17 Koerper, M,; Dallman, P.: "Serum iron concentration and transferrin saturation in the diagnosis of iron deficiency in children: Nomal developmental changes". J. Pediat. 91: 870, 1977.
${ }^{18}$ Soorinen, V.: Sümes, M.: "Developmental changes in serum iron, total iron binding capacity, and transferrin saturation in infancy". J. Pediat. 91: 875, 1977.

19 Cook, J.; Finch, C.: "Assesing iron status of a population”, Am. J. Clin. Nutr. 32: 2115, 1979.

20 Who Scientific group on Nutritional anemias, Wh. Hlth. Org. Techn. Rep. Ser. No 405, 1968.

21 Stekel, A, y Cols.: "Iron deficiency in infancy and childhood". Am. J. Clin. Nutr. 33: 110, 1980.

22 King. J.: De pablo, S.; Fontecilla, J.; Montes, F.: "Peroxidación de grasas de leche entera adicionada de hierro heminico". Rev. Ch. Nutr. Vol. 10, No2, 1982 . 